\title{
Research Paper: A Randomized Clinical Trial on the Effect of Biofeedback on Pain and Quality of Life of Patients With Chronic Coccydynia
}

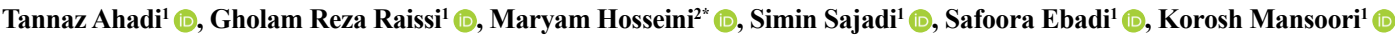 \\ 1. Neuromusculoskeletal Research Center, Firoozgar Hospital, Iran University of Medical Sciences, Tehran, Iran \\ 2. Department of Physical Medicine and Rehabilitation, Imam Khomeini Hospital, Tehran University of Medical Sciences, Tehran, Iran.
}

\begin{tabular}{|c|c|}
\hline \multirow{2}{*}{$\begin{array}{l}\text { Use your device toscan } \\
\text { and read the article online }\end{array}$} & \\
\hline & \\
\hline $4+44$ & \\
\hline 口rtits & dei) http://dx.doi.org/10.32598/ben.11.6.1553.1 \\
\hline
\end{tabular}

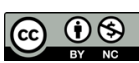

Article info:

Received: 16 Oct 2018

First Revision: 10 Dec 2018

Accepted: 02 Oct 2019

Available Online: 01 Nov 2020

Keywords:

Coccyx pain, Biofeedback, Pelvic floor, Quality of life

\begin{abstract}
AB S T RAC T
Introduction: Pelvic floor muscles dysfunction is one of the most important etiologies of coccydynia; therefore, manual therapies have been proposed as the first line of treatment. The purpose of this study was to investigate the effect of biofeedback as a new approach in the treatment of coccydynia.

Methods: Thirty women were randomized into two groups. Both groups were injected with the corticosteroid. One group received pelvic floor muscle exercises plus biofeedback while the other group only performed exercises. The patient's pain was measured using the Visual Analog Scale (VAS) in the first visit and after 1,2, and 6 months of follow-up as well as Dallas pain and SF-36 quality of life questionnaires before and 2 months after the treatment

Results: Pain had improved significantly after 1,2, and 6 months in both groups compared to the baseline. However, the amount of change was not different between the groups at any time interval. The results were the same for the Dallas pain scale and SF-36 quality of life questionnaire.

Conclusion: Adding biofeedback to pelvic floor muscle exercises did not lead to any further improvement in the management of chronic coccydynia. Further studies with larger sample
\end{abstract} sizes may reveal the effect of biofeedback more clearly.

\section{* Corresponding Author:}




\section{Highlights}

- We report on the effect of pelvic floor muscles biofeedback in the treatment of coccydynia for the first time.

- Coccydynia significantly affects patients' function and quality of life without any accepted treatment.

- According to the reputable theory of pelvic floor muscle dysfunction as one of the most important etiologies of coccydynia, manual therapies have been proposed as the first line of treatment.

- Pelvic floor muscles biofeedback would be a new and safe treatment option in this area.

- The paper should be of interest to readers in the areas of the importance of the issue and the novelty of the treatment approach.

\section{Plain Language Summary}

Coccydynia is a painful condition that is felt in the coccyx area. Its prevalence in women is five times more than in men. The most common type of trauma is falling back in a sitting position. Repeated minor trauma for example due to prolonged sitting in an inappropriate position is also mentioned as an important cause of coccydynia. Biofeedback is a method of gaining control and strengthening the muscles. In this study, we aimed to investigate the effect of adding pelvic floor biofeedback to topical anti-inflammatory drug injection plus pelvic floor muscle exercises in patients with coccydynia. In the end, we have concluded that adding biofeedback to pelvic floor muscles exercises did not lead to any further improvement in pain and quality of life in patients with chronic coccydynia.

\section{Introduction}

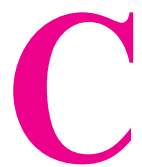

occydynia (also referred to as coccygodynia) is a painful condition that is felt in the coccyx area and is a symptom more than a distinct disease (Patijn et al., 2010). Its prevalence in women is five times more than in men due to the risk of birth trauma as well as the longer and more posterior position of the bone. Besides, there is a direct correlation between body mass index (BMI) and the risk of coccydynia. BMI greater than $27.4 \mathrm{~kg} / \mathrm{m}^{2}$ in women and $29.4 \mathrm{~kg} / \mathrm{m}^{2}$ in men increases the risk of its development (Patijn et al., 2010).

Most cases have abnormal mobility of coccyx in dynamic radiographs and many of them have a history of a primary trauma (Fogel, Cunningham III, \& Esses, 2004; Grgić, 2011; Patel, Appannagari, \& Whang, 2008). The most common type of trauma is falling back in a sitting position. Repeated minor trauma or overloading (for example due to prolonged sitting in an inappropriate position, cycling, etc.) are also mentioned as important causes of coccydynia. Other suggested etiologies are degenerative changes in the sacrococcygeal and intercoccygeal joints, spicule, osteomyelitis, tumors, and so on
(Grgić, 2011). Besides, coccydynia can occur due to hypertonicity of pelvic floor muscles (Nelson et al., 2012).

A key finding in the diagnosis of suspected patients is tenderness in direct touching of the coccyx area, pubococcygeal, and sacrococcygeal ligaments. Examination of ligaments, muscles, including levator ani and gluteus maximus, and joints near the tailbone is also very important (Frontera, Silver, \& Rizzo Jr, 2014).

For the first time in 1837, Simpson stated that coccydynia is related to coccygeal injury with the inflammation of surrounding tissues and pelvic floor muscle spasm (Scott, Fisher, Bernstein, \& Bradley, 2017). Based on Simpson's theory, after tailbone trauma and inflammation, any contraction in muscles attached to the coccyx would result in pain. In 1937, Thiele reported 80 patients with coccydynia and or pain in the supragluteal or posterior thigh region who were treated with pelvic muscles massage. Of those, $60 \%$ were completely cured, $33.7 \%$ got markedly better and only $6.6 \%$ did not change. He proposed that pelvic floor muscles are the primary etiology of coccydynia (Chiarioni, Nardo, Vantini, Romito, \& Whitehead, 2010).

Since the statement of Thiele and Simpson's hypothesis about the involvement of pelvic floor muscles as the ma- 
jor cause in coccydynia, many studies have demonstrated the presence of spasm and myofascial trigger points of these muscles in patients with coccygeal pain. Associated symptoms such as dyspareunia and pain during defecation which are seen in some patients are also related to pelvic floor muscles involvement (Scott et al., 2017).

To date, there has been little agreement on the definite treatment for coccydynia (De Andrés \& Chaves, 2003; Hodges, Eck, \& Humphreys, 2004). The currently available treatments include conservative approaches (such as rest and ring-shaped cushions), medication, including NSAIDs, physiotherapy, acupuncture, manual therapies, and interventional methods such as injection of local anesthetic and corticosteroid in the painful structures, intradiscal injection, radiofrequency destruction of coccygeal disks, caudal and impar ganglion block. In refractory cases, partial or total coccygectomy is performed (De Andrés \& Chaves, 2003; Fogel et al., 2004; Grgić, 2011; Patijn et al., 2010; Zayer, 1996).

The most recently introduced form of protective padding is a "wedge-shaped cushion" or "coccygeal cushion". It can efficiently decrease pressure on the tailbone in a sitting position (Lirette, Chaiban, Tolba, \& Eissa, 2014). Conservative therapies are the first-line in the treatment of coccydynia, including manual therapies such as levator ani muscle massage, sacrococcygeal and intercoccygeal joints mobilization and manipulation (Baloch, Hashmi, Khalid, \& Hashmi, 2012).

Local steroid injection in patients who do not respond to supportive therapies is useful and is the treatment option that should be tested pre-surgically. However, local injection of steroids is also accepted as the first-line treatment of coccydynia (Baloch et al., 2012; Finsen, 2001; Scott et al., 2017). Most patients need reinjection over time. The effect of corticosteroid injection has been reported between $60 \%$ to $75 \%$, although the data are often obtained from case reports or small case series (Scott et al., 2017).

The new guidelines are in favor of conservative therapies. The new review articles encourage practitioners to use a combination of manual mobilization of sacrococcygeal joint capsule with a local injection of steroids and anesthetics. Due to unpredictable long-term consequences and the risk of severe complications, surgical treatment is recommended only in refractory cases (Scott et al., 2017). According to the theory of pelvic floor muscle involvement as a major cause of coccydynia, pelvic floor biofeedback can be considered as a treatment option.
EMG biofeedback is a method of gaining control and strengthening the muscles. In this method, signals of muscle electrical activity are recorded via special sensors and converted to visual and auditory cues, which are used to identify and control muscle activity. In this manner, weak muscles are activated as needed and hypertonic muscles are relaxed. The overall result is the improvement of the coordination of muscle activity. This technique enables the patient to take over the voluntary contraction of muscles (Traycoff, Crayton, \& Dodson, 1989).

For the first time in 1974, the biofeedback of pelvic floor muscles was introduced for the treatment of patients with fecal incontinence and the treatment results were successful. Since then, this method was used in the treatment of constipation and pelvic organ prolapse-related disorders such as urinary incontinence and sexual problems. Application of this method in the management of pelvic pain such as chronic pelvic pain syndrome, rectal and perineal pain dates back to recent years. The results of the published studies have shown that biofeedback is effective in the treatment of patients with chronic pelvic pain. Compared to other treatments such as medication or surgery, biofeedback has no side effects and there is no report of aggravating symptoms (Wexner \& Stollman, 2006).

In a study that was conducted on 79 patients with coccydynia between 2009 and 2012, the study patients were treated with physical therapy, emphasizing pelvic floor muscle relaxation. The overall percentages of improvement within 4 weeks of follow-up were reported to be $71.9 \%$ (Scott et al., 2017). Another study in 2009 on 157 patients with levator ani syndrome was performed to compare the effects of treatment with biofeedback, electrogalvanic stimulation, and massage. The results showed that biofeedback was more successful than the other two methods (Chiarioni et al., 2010).

Given the high prevalence of patients with coccydynia and the undeniable effect of this pathology on the patients' quality of life and also due to the positive results of biofeedback in chronic pelvic pain and the proven superiority of supportive therapy in the treatment of coccydynia, this study aimed to investigate the effect of adding biofeedback therapy to topical steroid injection plus pelvic floor muscle exercises in patients with coccydynia in a randomized controlled trial.

\section{Methods}

This is a single-blinded clinical trial conducted on 30 patients with coccydynia, referred to the clinic of Physical Medicine and Rehabilitation from May 2015 to May 2016. 
The inclusion criteria were 18-65 years old literate females with VAS score equal to or greater than 4 in each one of the sitting, standing, or supine positions for at least 2 months, no history of coccygeal injection or manipulation during the last two months, no systemic diseases (poor control diabetes, rheumatoid arthritis, etc.), no history of malignancy, coccygeal dislocation, acute fissure, no skin problem at the injection site and no simultaneous biofeedback therapy for the treatment of other pelvic floor disorders.

The exclusion criteria were patients not willing to continue the study anymore and noncompliance to any one of the inclusion criteria during the trial. This study was approved by the Ethics Committee of Iran University of Medical science and registered by the Iranian Registry of Clinical Trials (IRCT) with number 2016011824621N4.

\subsection{Study design}

Before entering the trial, the study design was described to all patients. Also, local injection side effects and the approach to manage and treat these complications were explained. The information, including gender, age, pain duration, body mass index, and presence or absence of a specific trauma before pain initiation was collected. Also, pain in sitting, standing, and supine positions was registered via VAS ruler.

In all patients, tenderness on the tailbone area was confirmed through the surface and intra-anal examination. In addition, lateral dynamic x-rays were taken to investigate the cause of coccydynia. The patients were randomized into two groups of 15 based on baseline pain intensity (VAS score $\geq 7$ or VAS $<7$ ).

In both groups in the initial visit, after history taking, physical examination, and radiologic assessment, topical steroid injection was done in the following way. After prepping, local anesthesia was performed with $2 \mathrm{~mL}$ of $2 \%$ lidocaine with a 22 -gauge needle. Then, $1 \mathrm{~mL}$ of 40 $\mathrm{mg}$ methylprednisolone was injected at the point of maximum tenderness. It was recommended using 5 minutes of cold compress within every 6 hours at home and avoiding weight bearing on the tailbone for at least 48 hours. After these 48 hours, the patient should use wedge-shaped cushions for prolonged sitting.

After 48 hours from the injection, the patients in both groups were asked to do pelvic floor muscle exercises (Kegel). Besides, the patients in the intervention group have undergone 8 sessions of biofeedback therapy.
Treatment follow-ups were done once at the end of 4 weeks, after completing the biofeedback sessions, at the end of 8 weeks, and finally 24 weeks after starting the treatment in both groups with the assessment of VAS score in sitting position. Also, the Dallas pain questionnaire and SF36 quality of life scale were filled before the treatment and 8 weeks after starting the treatment.

\subsection{Teaching pelvic floor muscles strengthening ex-} ercises

After explaining the anatomy of the pelvis, Pelvic Floor Muscle (PFM) function, and the importance of exercise and its continuity to strengthen the pelvic floor muscles by the physical medicine and rehabilitation resident to the patient, the correct form of PFM contraction was instructed and practiced. In this way, the patients were asked to lie in lateral decubitus position, while hip and knee were flexed and abdominal and gluteal muscles were relaxed and to breathe slowly and naturally. Then, just to learn how to exercise correctly, after wearing gloves, the patient puts her fingers on the anal outlet and tries to pull in her fingers with pelvic floor muscle contraction, the so-called squeezing. By doing so, the patient became aware of the pelvic floor upward movement. Then the patient was asked to relax her muscles and so pelvic floor again returns to its former state. During these contractions, other muscles such as abdominal and gluteal muscles should be relaxed. After learning the correct contraction, the patient was instructed to exercise 5 times a day, each time performing 10 voluntary maximum contractions, maintain each contraction for 5 seconds with $10 \mathrm{sec}-$ onds of rest intervals. The exercise was done in the following order: lateral decubitus position, sitting, and standing. A table diary was given to record the workouts to increase patient compliance. In addition to all the above instructions, patients in the intervention group were trained for 4 weeks and a total of eight 30-minute sessions (twice a week) with pressure biofeedback by a trained physiotherapist.

\subsection{Biofeedback protocol}

Enraf-Nonius (Myomed632x- Poland 2000) was used to deliver biofeedback, beginning with the patient in a sidelying position, and the probe fixed intrarectally. Three contractions were recorded and the average contraction pressure, rest pressure, and maximum contraction pressure were determined. Then, according to the analysis of the contraction parameters, proper strengthening or relaxation exercises were prescribed. The patient continued exercises between and after the sessions. 


\subsection{Outcome measures}

Pain in sitting, standing, and supine positions were measured using a VAS ruler which is a simple instrument containing 10 scores between 0 and 10 in which the 0 refers to no pain and 10 is the worst intolerable pain (Gould, Kelly, Goldstone, \& Gammon, 2001).

Dallas pain scale is a self-report questionnaire which is widely used since the 1990s because of its simplicity and short administration time (about 5 minutes). It evaluates the functional impact of pain on 4 aspects of the patient's life. These 4 sections are daily activities, work/leisure activities, anxiety/depression, and social interests (Marty et al., 2016).

The short-form health survey is a 36-item, self-reported survey of patient's health. It has 8 parts of vitality, physical functioning, bodily pain, general health perceptions, physi- cal role functioning, emotional role functioning, social role functioning, and mental health. Each part has a specific score and also a total score is calculated as the mean of all sections. The Persian version of this questionnaire was validated in 2004 by Montazeri et al. (Montazeri, Goshtasebi, Vahdaninia, \& Gandek, 2005).

\subsection{Statistical analysis}

The obtained data were analyzed using SPSS V. 22. The Kolmogorov-Smirnov test showed that the data were normally distributed. Mixed ANOVA was used to explore the interaction effect of time and group on outcome measures. The independent $t$ test and Mann-Whitney $U$ tests were used to compare parametric and non-parametric data at baseline, respectively.

Table 1. Patients' baseline characteristics (Each group: $\mathrm{n}=15$ )

\begin{tabular}{|c|c|c|c|c|}
\hline & \multirow{2}{*}{ Group } & \multicolumn{2}{|c|}{ Mean士SD } & \multirow{2}{*}{$\mathbf{P}$} \\
\hline & & Biofeedback & Exercise & \\
\hline Age & - & $41.47 \pm 8.96$ & $35.60 \pm 10.81$ & 0.117 \\
\hline BMI & - & $26.68 \pm 3.818$ & $26.42 \pm 5.003$ & 0.784 \\
\hline Pain duration & - & $37.80 \pm 45.52$ & $37.33 \pm 59.13$ & 0.467 \\
\hline \multirow[t]{4}{*}{ VAS } & - & $8.13 \pm 1.64$ & $7.87 \pm 1.18$ & 0.669 \\
\hline & Dallas (daily activities) & $38.40 \pm 15.13$ & $40.80 \pm 14.46$ & 0.661 \\
\hline & Dallas (work/leisure activities) & $58.33 \pm 28.26$ & $48.67 \pm 19.40$ & 0.284 \\
\hline & \multicolumn{4}{|c|}{ Pain } \\
\hline & Dallas (anxiety/depression) & $41.00 \pm 25.50$ & $44.00 \pm 20.65$ & 0.711 \\
\hline & Dallas (social interest) & $24.67 \pm 25.52$ & $30.33 \pm 17.64$ & 0.509 \\
\hline \multirow{9}{*}{ Quality of Life } & Physical functioning & $67.33 \pm 18.30$ & $70.00 \pm 19.54$ & 0.751 \\
\hline & Role physical & $35.00 \pm 43.09$ & $30.00 \pm 31.62$ & 0.720 \\
\hline & Role emotional & $51.11 \pm 45.19$ & $42.22 \pm 46.23$ & 0.599 \\
\hline & Energy/fatigue & $55.66 \pm 21.36$ & $39.00 \pm 15.82$ & 0.021 \\
\hline & Emotional well-being & $51.76 \pm 23.62$ & $55.53 \pm 16.54$ & 0.273 \\
\hline & Social functioning & $55.83 \pm 25.81$ & $63.33 \pm 21.37$ & 0.394 \\
\hline & Pain & $35.16 \pm 19.12$ & $37.66 \pm 16.24$ & 0.702 \\
\hline & General health & $50.16 \pm 20.27$ & $53.00 \pm 12.92$ & 0.652 \\
\hline & Total & $63.86 \pm 25.69$ & $48.84 \pm 15.49$ & 0.644 \\
\hline
\end{tabular}




\section{Results}

Forty patients were examined by the specialist. Six patients were not eligible for the study, so a total of 34 patients with chronic coccydynia (at least 2 months of pain) were enrolled. During the study, 2 patients in the biofeedback group were not satisfied with treatment results and received complementary treatments. Also, two patients in the exercise group did not complete the follow-up sessions and refused further treatment due to personal reasons. Finally, data from 30 patients ( 15 in each group) were analyzed. The flow diagram of participants is shown in Figure 1.

All participants were females. Their average age was 41.4 years $(\mathrm{SD}=8.96)$ in the biofeedback group and 35.6 years $(\mathrm{SD}=10.8)$ in the exercise group. The average BMI in both groups was $26 \mathrm{~kg} / \mathrm{m}^{2}(\mathrm{SD}=4)$ and each patient had suffered coccydynia for approximately three years $(\mathrm{SD}=45.5$ months for biofeedback and 59.1 for exercise groups) before starting the treatment. Analysis of the demographic data indicated that the two groups were not significantly different in terms of age, body mass index, and duration of pain before the treatment $(\mathrm{P}>0.05)$.

The mean baseline VAS score was $8.13(\mathrm{SD}=1.64)$ in the biofeedback group and $7.87(\mathrm{SD}=1.18)$ in the exercise group. VAS and Dallas scores (in any of the four domains) did not differ significantly at baseline between the groups (Table 1).

Regarding the SF-36 quality of life questionnaire, the initial scores had no significant difference between the two

Table 2. Within groups differences of VAS, Dallas, and SF-36 QOL scores

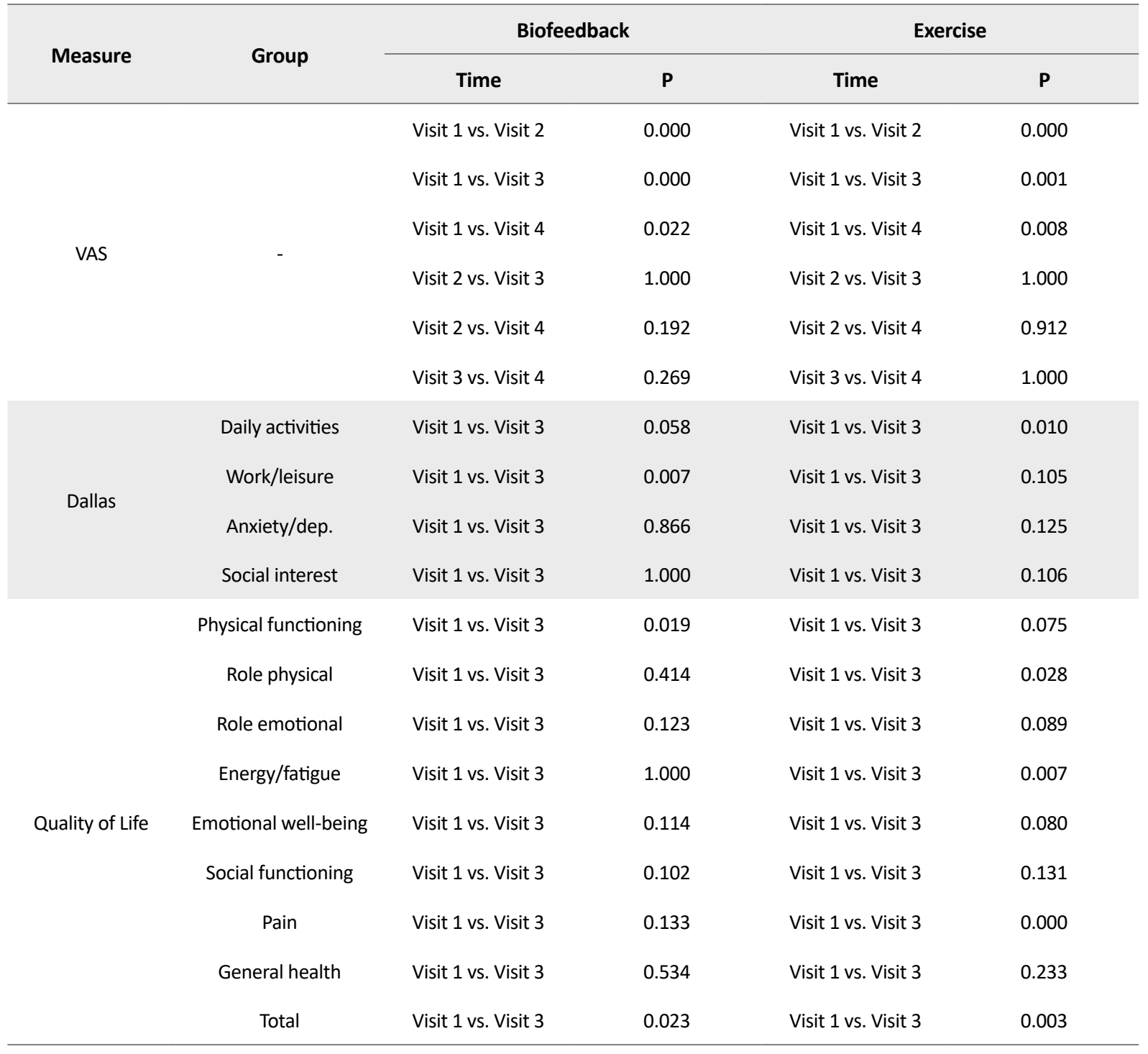


Table 3. Interaction effect of time and group on VAS, Dallas, and SF36 QOL scores

\begin{tabular}{|c|c|c|c|c|c|c|c|}
\hline & Measure & Time & Mean Square & $\mathbf{F}$ & df & Error & $\mathbf{P}$ \\
\hline \multirow{6}{*}{ VAS } & \multirow{6}{*}{ - } & Visit 1 vs. Visit 2* & 0.53 & 0.08 & 1 & 28 & 0.775 \\
\hline & & Visit 1 vs. Visit 3 & 4.03 & 0.55 & 1 & 28 & 0.462 \\
\hline & & Visit 1 vs. Visit 4 & 0.53 & 0.08 & 1 & 28 & 0.774 \\
\hline & & Visit 2 vs. Visit 3 & 1.63 & 0.89 & 1 & 28 & 0.352 \\
\hline & & Visit 2 vs. Visit 4 & 2.13 & 0.30 & 1 & 28 & 0.583 \\
\hline & & Visit 3 vs. Visit 4 & 7.50 & 0.79 & 1 & 28 & 0.381 \\
\hline \multirow{4}{*}{ Dallas } & Daily activities & Visit 1 vs. Visit 3 & 43.20 & 0.73 & 1 & 28 & 0.117 \\
\hline & Work/leisure & Visit 1 vs. Visit 3 & 653.33 & 0.29 & 1 & 28 & 1.123 \\
\hline & Anxiety/dep. & Visit 1 vs. Visit 3 & 163.33 & 0.65 & 1 & 28 & 0.207 \\
\hline & Social interest & Visit 1 vs. Visit 3 & 480.00 & 0.21 & 1 & 28 & 1.622 \\
\hline \multirow{9}{*}{ Quality of Life } & Physical functioning & Visit 1 vs. Visit 3 & 13.33 & 13.33 & 1 & 28 & 0.818 \\
\hline & Role physical & Visit 1 vs. Visit 3 & 240.83 & 240.83 & 1 & 28 & 0.677 \\
\hline & Role emotional & Visit 1 vs. Visit 3 & 333.33 & 333.33 & 1 & 28 & 0.607 \\
\hline & Energy/fatigue & Visit 1 vs. Visit 3 & 907.50 & 907.50 & 1 & 28 & 0.132 \\
\hline & Emotional well-being & Visit 1 vs. Visit 3 & 50.70 & 50.70 & 1 & 28 & 0.645 \\
\hline & Social functioning & Visit 1 vs. Visit 3 & 1165.63 & 1165.63 & 1 & 28 & 0.112 \\
\hline & Pain & Visit 1 vs. Visit 3 & 130.20 & 130.20 & 1 & 28 & 0.616 \\
\hline & General health & Visit 1 vs. Visit 3 & 25.20 & 25.20 & 1 & 28 & 0.801 \\
\hline & Total & Visit 1 vs. Visit 3 & 70.71 & 70.71 & 1 & 28 & 0.566 \\
\hline
\end{tabular}

NEURSCIENCE

groups in any of the areas of health, except for the energy/ fatigue area (Table 1). The mean VAS pain scores noticeably improved after treatment in both groups in 1,2 , and 6 months of follow-up $(\mathrm{P}<0.05$, Table 2); however, the amount of change was not different between the two groups at any time interval $(\mathrm{P}>0.05$, Table 3$)$.

Besides, within-group improvements occurred in Dallas pain scale and SF-36 quality of life questionnaire as follows: for Dallas pain questionnaire in the area of work/ leisure activities in the exercise group and daily activities in the biofeedback group; for SF-36 quality of life questionnaire in physical functioning and total scores in the biofeedback group and domains of physical health, energy/fatigue, pain, and the total scores in the exercise group $(\mathrm{P}<0.05$, Table 2). However, there was no interaction between time and group in any of the areas two months after the intervention (Table 3).

\section{Discussion}

According to the current literature, this is the first study to investigate the effect of biofeedback in the management of coccydynia in a randomized clinical trial design. In recent years, many studies have been conducted on conservative treatments for coccydynia, such as shockwave therapy, pelvic floor muscle massage, coccygeal manipulation, and mobilization, but no study has focused on pelvic floor muscle biofeedback. This is a new approach for achieving conscious control in dysfunctional muscles.

In the present study, steroid injection and pelvic floor exercises were applied for both groups. Besides, 8 sessions of biofeedback were also added to the treatment regimen in the intervention group. The results showed that in both groups, the VAS score dropped significantly (44\% decrease in the biofeedback group and $42 \%$ in the exercise group af- 


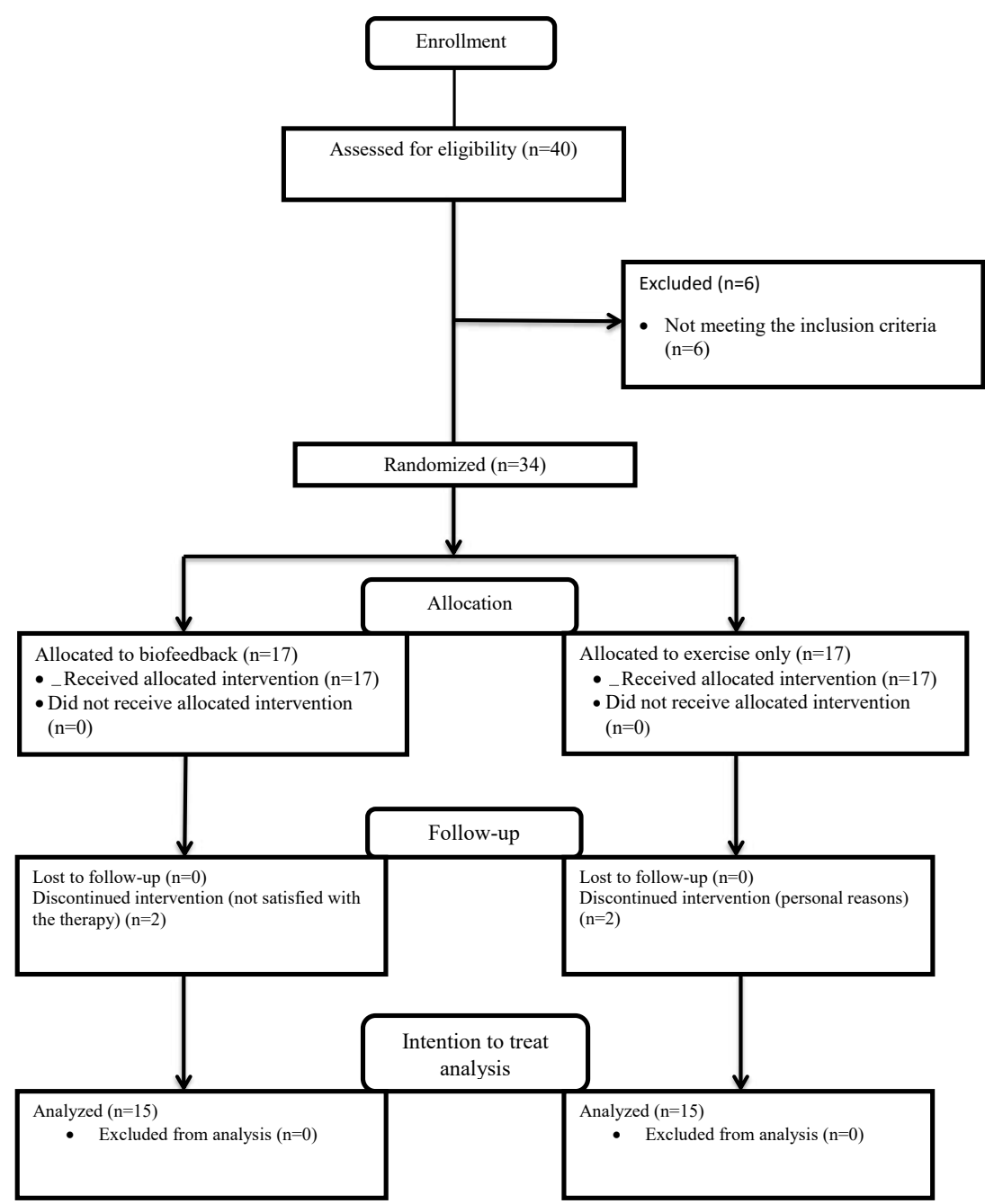

Figure 1. Flowchart of the participants

ter 4 weeks). This significant improvement in pain was seen in all the follow-up measurements in both the intervention and control groups.

Despite the positive results and remarkable intra-group changes, there was no significant statistical difference between the two groups in terms of the amount of pain reduction measured by the Visual Analog Scale (VAS). It would convey that, adding biofeedback to the exercise regimen did not lead to any further improvement in pain. The positive outcomes of both treatment regimens remained up to 6 months after the intervention.

These results were in line with Kelly M. Scott and his colleagues' study in the field of pain reduction using pelvic floor physical therapy and muscle training. In this retrospective chart review conducted on 79 patients with chronic coccydynia and post-coccygectomy pain, the participants underwent pelvic floor physical therapy with an emphasis on the relaxation of the pelvic floor muscles. For this purpose, specific exercises, anal or vaginal dilators, muscle energy techniques to correct pelvic girdle alignment, and if necessary, biofeedback with surface electrodes and sometimes rectal or vaginal probes were used to release pelvic floor muscles. Secondary treatments involving administration of baclofen, impar ganglion block, and injection of the coccygeus muscle trigger points were delivered in several patients. The average pain score decreased from 5.08 to $1.91(\mathrm{P}<0.001)$ and it reduced among postsurgical patients from 6.64 to 3.27 as well. Overall, $71.9 \%$ improvement within 4 weeks after treatment was reported (Scott et al., 2017).

The general approach in this study was pelvic floor physical therapy. As it was a retrospective study, the treatment protocol was not identical for all patients and each patient was managed according to his/her basic problems. Followup duration was short (4 weeks) and there was no control group to compare. Considering all the aspects involved in 
coccydynia, including musculoskeletal structures was the point of strength in this study.

Coccydynia is a multidimensional pathology in which all the surrounding structures such as pelvic organs, muscular and bony elements can be responsible as pain triggers. A comprehensive exam of the lumbosacral spine and pelvic girdle such as the pelvic diaphragm, gluteal muscles, sacroiliac, and hip joints as well as visceral elements, including rectal and vaginal exam is necessary to set a treatment plan.

In this study, biofeedback with the contraction-relaxation technique was added to the specific exercises instructed to patients. However, its effect was not significantly different from pelvic floor muscle exercises alone. Given that more than $90 \%$ of patients admitted for treatment suffering pelvic floor muscle weakness (based on the average power of contraction reported by biofeedback device), both muscle strengthening and relaxation were the main goals of therapy sessions. Nevertheless, many studies in the field of pelvic floor rehabilitation mainly focus on relaxation techniques. For example in a study conducted by Chiarioni et al. in 2010 on 157 patients with levator ani syndrome (LVS), therapeutic effects of massage, biofeedback (relaxation training), and electrogalvanic stimulation (EGS) were compared with each other. The study patients were followed after treatment at intervals of 1 , $3,6,12$, and 24 months. Outcome measures were VAS score, number of days with pain per month, and frequency of defecation over the past week. Among patients with a high probability of LVS, $87 \%$ in biofeedback, $45 \%$ in EGS, and $22 \%$ in the massage group reported significant pain relief. Remission remained for 24 months. Finally, it was concluded that biofeedback and EGS were effective in the treatment of LVS by increasing the patient's ability to relax the pelvic floor muscles and increasing the pain threshold and better effects were seen in the field of biofeedback (Chiarioni et al., 2010).

Although the exact pathophysiology of LVS is unknown, pelvic floor muscle spasm has been recognized as the most likely cause. In some resources, coccydynia and LVS are considered as variants of a single pathology. In this study, biofeedback was more effective compared to electrical stimulation and massage. In the present study, however, no further improvement was seen after adding biofeedback to the exercises. Fewer sample size ( $n=30$ vs. $n=157)$ may be an explanation for this difference. Perhaps, by increasing the sample size the results will become closer to each other. Also, it is assumed that the correct training of pelvic floor exercises would result in the correct performance of the patient which may make exercise more efficacious.
One main goal in using biofeedback is accurate training of muscle contraction and relaxation through auditory and visual signals. In this study, exercise training was managed by a skilled therapist and any mistake was modified during the first visit to make patients implement the technique properly and this could have resulted in positive outcomes and successful pain control in the exercise therapy group.

Biofeedback is one of the common and useful methods in the treatment of urinary, gastrointestinal, and sexual disorders related to pelvic floor pathologies, including constipation, urinary, and fecal incontinence, and problems like dyspareunia and vaginismus. In a 4-year retrospective study conducted on 778 women, non-surgical treatments, including biofeedback were effective in the treatment of pelvic floor dysfunction, such as those related to defecation, urinary symptoms, and pelvic pain (Starr et al., 2013). In a clinical trial carried out in 2010 on 26 patients, it was found that biofeedback better improves bowel symptoms and anorectal function compared to standard therapies such as diet, exercise, and laxatives in patients with dyssynergic constipation (Rao, Valestin, Brown, Zimmerman, \& Schulze, 2010). In another study on 52 patients with fecal incontinence, biofeedback had positive results in improving patients' symptoms and quality of life (Leite \& Lacerda-Filho, 2013). Also, Ahadi et al. have concluded that patients with dyssynergic constipation with and without IBS will likely benefit from pelvic floor biofeedback (Ahadi et al., 2014).

Pathology in these disorders is often focused on the pelvic floor, including muscle weakness in functional disorders and incontinence or lack of coordination in their performance (dyssynergy) in obstructive disorders as well as muscle spasm in problems such as dyspareunia and vaginismus. Therefore, relying on techniques to strengthen weak muscles and relaxing hypertonic muscles will have a significant impact on the treatment.

In patients with coccydynia, we are encountered with mixed multidimensional pathophysiology which needs paying attention to all neighboring structures besides retraining of pelvic floor muscles with biofeedback.

On the other hand, pelvic floor muscle exercises alone have shown significant improvements in the management of patients with different types of pelvic floor dysfunction syndromes. In a systematic review published in Cochrane library in 2014 about the effect of Pelvic Floor Muscle Training (PFMT) for the treatment of urinary incontinence in women, the authors concluded that PFMT should be strongly recommended in the first-line conservative management plan for women with any type of urinary incon- 
tinence (Dumoulin, Hay-Smith, \& Mac Habée-Séguin, 2014). Besides, Brakken et al. proposed that pelvic floor muscle exercise was an efficacious treatment for sexual dysfunction in women with pelvic organ prolapse. The patients have reported increased self-confidence and more strength, control, and awareness of pelvic floor muscles (Brækken, Majida, Ellström Engh, \& Bø, 2015).

Pelvic floor muscle exercises were also shown successful results in the management of postpartum anal incontinence in an RCT conducted by Johannessen et al. (Johannessen, Wibe, Stordahl, Sandvik, \& Mørkved, 2017). The effectiveness of exercise in these studies and many others is what has been observed in the present study. It is assumed that precise exercise training and emphasis on contracting pelvic floor muscles instead of accessory muscles may cause this similarity.

In this study, the results of the Dallas pain scale were consistent with the VAS score, meaning that the amount of change analyzed by the interaction effect of time and group was not different between the two groups at any time interval while there were remarkable improvements in some mentioned areas within each group. It was also the same for the SF-36 QOL questionnaire. The level of overall improvement in the quality of life showed considerable promotion in both groups implying the effectiveness of exercise therapy alongside injection.

Study limitations were the small sample size and possible noncompliance of some patients in performing exercises at home and attending planned visiting sessions which led to dropping out of some participants and more lowering of the number of attendances.

As biofeedback has shown to be a safe and successful treatment in pelvic floor-related disorders, future research studies with larger sample size, more treating sessions, greater insist on pelvic floor relaxation techniques, and multidisciplinary approaches in the treatment of coccydynia are recommended. Adding biofeedback to the pelvic floor muscles exercises did not lead to any further improvement in pain and quality of life in patients with chronic coccydynia.

\section{Ethical Considerations}

\section{Compliance with ethical guidelines}

All study procedures involving human participants were in accordance with the ethical standards of the Institutional and or National Research Committee and with the 1964 Helsinki declaration and its later amendments or comparable ethical standards.

\section{Funding}

This research did not receive any grant from funding agencies in the public, commercial, or non-profit sectors.

\section{Authors' contributions}

All authors equally contributed to preparing this article.

\section{Conflict of interest}

The authors declared no conflict of interest.

\section{References}

Ahadi, T., Madjlesi, F., Mahjoubi, B., Mirzaei, R., Forogh, B., \& Daliri, S. S., et al. (2014). The effect of biofeedback therapy on dyssynergic constipation in patients with or without Irritable Bowel Syndrome. Journal of Research in Medical Sciences, 19(10), 950-5. [PMID] [PMCID]

Baloch, N. A., Hashmi, I., Khalid, M., \& Hashmi, M. (2012). Local infilteration of steroid in conjunction with coccygel manipulation as a treatment of coccydynia. Journal of Surgery Pakistan (International), 17(2), 65-7. http://old.jsp.org.pk/Issues/ JSP\%2017\%20(2)\%20April\%20-\%20June\%202012\%20PDF/ Muhammad\%20Khalid\%20OA.pdf

Brækken, I. H., Majida, M., Ellström Engh, M., \& Bø, K. (2015). Can pelvic floor muscle training improve sexual function in women with pelvic organ prolapse? A randomized controlled trial. The Journal of Sexual Medicine, 12(2), 470-80. [DOI:10.1111/jsm.12746] [PMID]

Chiarioni, G., Nardo, A., Vantini, I., Romito, A., \& Whitehead, W. E. (2010). Biofeedback is superior to electrogalvanic stimulation and massage for treatment of levator ani syndrome. Gastroenterology, 138(4), 1321-9. [DOI:10.1053/j.gastro.2009.12.040] [PMID] [PMCID]

De Andrés, J., \& Chaves, S. (2003). Coccygodynia: A proposal for an algorithm for treatment. The Journal of Pain, 4(5), 257-66. [DOI:10.1016/S1526-5900(03)00620-5]

Dumoulin, C., Hay-Smith, E. J. C., \& Mac Habée-Séguin, G. (2014). Pelvic floor muscle training versus no treatment, or inactive control treatments, for urinary incontinence in women. The Cochrane Database of Systematic Reviews, (5):CD005654. [DOI:10.1002/14651858.CD005654.pub3] [PMID]

Finsen, V. (2001). [Corticosteroid injection for coccygodynia (Norwegian)]. Tidsskrift for Den Norske Lægeforening, 121(24), 2832-3. [PMID]

Fogel, G. R., Cunningham III, P. Y., \& Esses, S. I. (2004). Coccygodynia: Evaluation and management. Journal of the American Academy of Orthopaedic Surgeons, 12(1), 49-54. [DOI:10.5435/00124635-200401000-00007] [PMID]

Frontera, W. R., Silver, J. K., \& Rizzo Jr, T. D. (2014). Essentials of physical medicine and rehabilitation. Elsevier Health Sciences. https://books.google.com/books?id=B0b-nQEACAAJ\&dq 
Gould, D., Kelly, D., Goldstone, L., \& Gammon, J. (2001). Examining the validity of pressure ulcer risk assessment scales: Developing and using illustrated patient simulations to collect the data INFORMATION POINT: Visual analogue scale. Journal of Clinical Nursing, 10(5), 697-706. [DOI:10.1046/j.1365-2702.2001.00525.x] [PMID]

Grgić, V. (2011). [Coccygodynia: Etiology, pathogenesis, clinical characteristics, diagnosis and therapy (Croatian)]. Liječnicki Vjesnik, 134(1-2), 49-55. [PMID]

Hodges, S. D., Eck, J. C., \& Humphreys, S. C. (2004). A treatment and outcomes analysis of patients with coccydynia. The Spine Journal, 4(2), 138-40. [DOI:10.1016/j.spinee.2003.07.011] [PMID]

Johannessen, H. H., Wibe, A., Stordahl, A., Sandvik, L., \& Mørkved, S. (2017). Do pelvic floor muscle exercises reduce postpartum anal incontinence? A randomised controlled trial. BJOG: An International Journal of Obstetrics and Gynaecology, 124(4), 686-94. [DOI:10.1111/1471-0528.14145] [PMID]

Leite, F. R., de Lima, M. J. R., \& Lacerda-Filho, A. (2013). Early functional results of biofeedback and its impact on quality of life of patients with anal incontinence. Arquivos de Gastroenterologia, 50(3), 163-9. [DOI:10.1590/S0004-28032013000200029] [PMID]

Lirette, L. S., Chaiban, G., Tolba, R., \& Eissa, H. (2014). Coccydynia: An overview of the anatomy, etiology, and treatment of coccyx pain. The Ochsner Journal, 14(1), 84-7. [PMID] [PMCID]

Marty, M., Courvoisier, D., Foltz, V., Mahieu, G., Demoulin, C., \& Gierasimowicz, A., et al. (2016). How much does the Dallas Pain Questionnaire score have to improve to indicate that patients with chronic low back pain feel better or well? European Spine Journal, 25(1), 304-9. [DOI:10.1007/s00586-015-3957-3] [PMID]

Montazeri, A., Goshtasebi, A., Vahdaninia, M., \& Gandek, B. (2005). The Short Form Health Survey (SF-36): Translation and validation study of the Iranian version. Quality of Life Research, 14(3) 875-82. [DOI:10.1007/s11136-004-1014-5] [PMID]

Nelson, P., Apte, G., Justiz, R., Brismeé, J. M., Dedrick, G., \& Sizer, P. S. (2012). Chronic female pelvic pain-part 2: Differential diagnosis and management. Pain Practice, 12(2), 111-41. [DOI:10.1111/ j.1533-2500.2011.00492.x] [PMID]

Patel, R., Appannagari, A., \& Whang, P. G. (2008). Coccydynia. Current Reviews in Musculoskeletal Medicine, 1(3-4), 223. [DOI:10.1007/ s12178-008-9028-1] [PMID] [PMCID]

Patijn, J., Janssen, M., Hayek, S., Mekhail, N., Van Zundert, J., \& Van Kleef, M. (2010). 14. Coccygodynia. Pain Practice, 10(6), 554-9. [DOI:10.1111/j.1533-2500.2010.00404.x] [PMID]

Rao, S. S., Valestin, J., Brown, C. K., Zimmerman, B., \& Schulze, K. (2010). Long-term efficacy of biofeedback therapy for dyssynergic defecation: Randomized controlled trial. The American Journal of Gastroenterology, 105(4), 890-6. [DOI:10.1038/ajg.2010.53] [PMID] [PMCID]

Scott, K. M., Fisher, L. W., Bernstein, I. H., \& Bradley, M. H. (2017). The treatment of chronic coccydynia and postcoccygectomy pain with pelvic floor physical therapy. PMER, 9(4), 367-76. [DOI:10.1016/j.pmrj.2016.08.007] [PMID]

Starr, J. A., Drobnis, E. Z., Lenger, S., Parrot, J., Barrier, B., \& Foster, R. (2013). Outcomes of a comprehensive nonsurgical approach to pelvic floor rehabilitation for urinary symptoms, defecatory dysfunction, and pelvic pain. Female Pelvic Medicine \& Reconstructive Surgery, 19(5), 260-5. [DOI:10.1097/SPV.0b013e31829cbb9b] [PMID]
Traycoff, R. B., Crayton, H., \& Dodson, R. (1989). Sacrococcygeal pain syndromes: Diagnosis and treatment. Orthopedics, 12(10), 1373-7. [PMID]

Wexner, S. D., \& Stollman, N. (2006). Diseases of the colon. Boca Raton: CRC Press. https://books.google.com/ books?id=shqgmAEACAAJ\&hl

Zayer, M. (1996). Coccygodynia. The Ulster Medical Journal, 65(1) 58-60. [PMID] [PMCID] 
This Page Intentionally Left Blank 